\title{
Saccharomyces cerevisiae SFP1: at the crossroads of central metabolism and ribosome biogenesis
}

\author{
Chiara Cipollina, ${ }^{1} \dagger$ Joost van den Brink, ${ }^{2}$ Pascale Daran-Lapujade, ${ }^{2,3}$ \\ Jack T. Pronk, ${ }^{2,3}$ Danilo Porro ${ }^{1}$ and Johannes $H$. de Winde ${ }^{2,3}$ \\ ${ }^{1}$ Dipartimento di Biotecnologie e Bioscienze, Università degli Studi di Milano-Bicocca, \\ P.za della Scienza 2, 20126 Milano, Italy
${ }^{2}$ Department of Biotechnology, Delft University of Technology, Julianalaan 67, 2628 BC Delft, The Netherlands
${ }^{3}$ Kluyver Centre for Genomics of Industrial Fermentation, Julianalaan 67, 2628 BC Delft, The Netherlands

Johannes H. de Winde

j.h.dewinde@tudelft.nl

\begin{abstract}
Saccharomyces cerevisiae SFP1 is required for nutrient-dependent regulation of ribosome biogenesis and cell size. A mutant deleted for SFP1 shows specific traits, including a slow growth phenotype, especially when growing on glucose. We recently analysed the physiology of an sfp $1 \Delta$ mutant and its isogenic reference strain in chemostat cultures. This approach was successful in revealing the effects of nutrients on the activity of Sfp1 independent of growth rate-related feedback. In the present work we exposed carbon-limited cultures of an $\operatorname{sfp} 1 \Delta$ mutant and its reference strain to sudden glucose excess. This allowed us to study the effect of SFP1 deletion on cell physiology when the cells are forced to exploit their maximum growth potential; this is similar to what happens in shake-flask cultures but with no bias due to growth rate differences. We show that nutrients differentiallly affect the role of Sfp1 in cell-size modulation and in transcriptional control. Furthermore, we report that while Sfp1 is necessary for the efficient glucose-dependent regulation of ribosome biogenesis genes, it is not required for the proper induction of ribosomal protein genes in response to glucose excess. Finally, our data suggest a role for Sfp1 in the regulation of glycolysis, further underlining its involvement in the network that links ribosome biogenesis and cell metabolism.
\end{abstract}

Received 7 February 2008

Revised 7 March 2008

Accepted 7 March 2008

\section{INTRODUCTION}

tPresent address: Department of Biotechnology, Delft University of Technology, Julianalaan 67, 2628 BC, Delft, The Netherlands.

Abbreviations: $\mathrm{CmM}, \mathrm{mM}$ carbon (content); FDR, false discovery rate; MIPS, Munich Institute for Protein Sequences; RP, ribosomal protein; SAM, significance analysis of microarrays.

The array data discussed in this publication have been deposited in the NCBI Gene Expression Omnibus (GEO; http://www.ncbi.nlm.nih.gov/ geo/) and are accessible through GEO series accession number GSE9644.

Three supplementary tables, listing genes showing significantly different expression in the sfp1 null mutant compared with the reference strain during ethanol/glucose-limited growth, significant differences in the transcriptional response between the reference and the mutant strain following the glucose pulse, and genes in particular clusters belonging to specified functional categories, and five supplementary figures, showing the cell-size distributions, extracellular glucose concentration and specific activity of glycolytic enzymes of the two strains, the $Z$ score profiles of the 12 clusters showing differential expression, and modulation of the budding index and cell concentration after the glucose pulse, are available with the online version of this paper.
The budding yeast Saccharomyces cerevisiae has evolved to survive sudden changes in the environment resulting from natural fluctuations in nutrient availability, temperature, osmotic pressure and other factors (Alberghina et al., 1998; Gasch \& Werner-Washburne, 2002; Kresnowati et al., 2006). As a consequence, S. cerevisiae can rapidly adjust its physiology in response to most different external perturbations. A number of pathways exist in yeast which successfully sense external conditions and generate a signal that is transmitted from the cell surface to the target biosynthetic machineries (Crespo \& Hall, 2002; Estruch, 2000; Rolland et al., 2002). Besides external stimuli, metabolic activity itself is an important player in the generation of such signals (Newcomb et al., 2003; Rolland et al., 2002).

In budding yeast, the TOR and PKA kinases are the major upstream regulators for the control of growth, metabolism and cell cycle progression in response to nutrients (Crespo 
\& Hall, 2002; Rolland et al., 2002). One of the most important targets controlled by TOR and PKA is the ribosome biosynthesis machinery (Klein \& Struhl, 1994; Martin et al., 2004; Powers \& Walter, 1999; ZuritaMartinez \& Cardenas, 2005). During optimal growth, a yeast cell spends most of its energy in making ribosomes. All three RNA polymerases are involved in this process and the activity of the RNA polymerase I, responsible for the transcription of the rRNA, accounts for up to $60 \%$ of the transcriptional activity of a growing cell (Warner, 1999). Ribosomal protein (RP) mRNAs monopolize the cell translational capacity during fast proliferation, and a network of more than 100 factors (encoded by the so called RiBi gene cluster) is required for the correct assembly of the mature ribosomal particles (Fatica \& Tollervey, 2002; Jorgensen et al., 2002). Such a massive utilization of cell resources explains why the control of ribosome biogenesis is a key issue for the economy of the cell. This is indeed finely tuned depending on the nutritional/environmental conditions (Martin et al., 2004; Zurita-Martinez \& Cardenas, 2005).

The control of rRNA, RP and RiBi gene expression by TOR and PKA occurs via parallel and partially redundant pathways within a coordinated regulatory network (Crespo \& Hall, 2002; Zurita-Martinez \& Cardenas, 2005). Although the topology of this network has not yet been fully elucidated, in recent years some important downstream targets, such as Fhl1, Sch9, Hmol and Sfp1, have been discovered (Berger et al., 2007; Jorgensen et al., 2002; Martin et al., 2004; Urban et al., 2007).

Sfp1 is a zinc finger protein for which a role in the control of RiBi and RP gene expression in response to nutrient availability has been proposed (Fingerman et al., 2003; Jorgensen et al., 2004; Marion et al., 2004). The localization and activity of Sfp1 appear to be modulated by TOR via a PKA-dependent and a PKA-independent pathway (Marion et al., 2004). It has been suggested that a condition of respiro-fermentative growth on glucose stimulates Sfp1 activity (Cipollina et al., 2005). Despite extensive research, the mechanisms of action and regulation of $S f p 1$ remain elusive, and the identification of its specific transcriptional targets is still controversial (Cipollina et al., 2008; Fingerman et al., 2003; Jorgensen et al., 2004; Marion et al., 2004).

Besides its role in ribosome biogenesis, Sfp1 is involved in the modulation of cell-size setting; a mutant deleted for SFP1 is characterized by a carbon source-dependent small size (whi) phenotype (Cipollina et al., 2005; Jorgensen et al., 2002). This dual role has led several researchers to suspect Sfp1 to be at the interface between ribosome biogenesis and condition-dependent cell-size control. Whether the setting of cell size is a readout of the growth events or is dictated upstream by ribosome biogenesis still remains an open question (Jorgensen et al., 2002; Schneider et al., 2004).

Most of the current evidence on the possible function of SFP 1 has so far been derived from the comparison of $s f p 1 \Delta$ mutants with their isogenic reference strains growing in shake flasks. However, the mutant strain has a slow growth phenotype which hinders the separation of the direct effects due to SFP1 deletion from secondary growth raterelated effects (Cipollina et al., 2005; Fingerman et al., 2003; Jorgensen et al., 2004; Marion et al., 2004).

We recently grew an $s f p 1 \Delta$ strain and its isogenic reference strain at the same specific growth rate by using chemostat cultures (Hoskisson \& Hobbs, 2005; Novick \& Szilard, 1950). Chemostat cultivation has proved to be a powerful tool to study the involvement of Sfp1 in cell-size modulation and transcriptional control in response to nutrients, independent of the growth rate-related phenotype (Cipollina et al., 2008).

Nevertheless, this chemostat-based approach suffers from several shortcomings. For instance, to maintain steady growth in chemostats, the growth rates are slower than the maximum specific growth rates $\left(\mu_{\max }\right)$ of the strains. In addition, the cultures were run under carbon limitation with slow feeding rates, leading to relatively slow in vivo carbon fluxes. It could therefore be surmised that the effect of the deletion of SFP1 on S. cerevisiae physiology would be weaker under these conditions than when the cells have to exploit their full potential for fast growth and metabolism (as in exponential growth in shake-flask cultivation). A new set of experiments was therefore designed to circumvent these drawbacks while preventing growth-rate bias.

In the present work, $s f p 1 \Delta$ and its reference strain cultivated at the same growth rate in aerobic carbonlimited chemostats were exposed to a sudden increase in glucose concentration coupled with a switch to anaerobiosis. Using this experimental set-up we explored the role of Sfp1 when cells have to sense the change in nutrient abundance and rapidly adjust their metabolism and growth to this new condition, exploiting their maximum growth potential. The study of the early response following glucose addition allowed us to quantify the effect of SFP1 deletion on cell physiology, cell-size control and transcriptional regulation, with no bias due to the slow-growth phenotype. Our results shed new light on the role of Sfp1 at the interface between nutrient sensing, ribosome biogenesis, size setting and carbon metabolism.

\section{METHODS}

Strains and growth conditions. The prototrophic reference $S$. cerevisiae strain CEN.PK113.7D (MATa MAL2-8c SUC2) and the isogenic strain CEN.PK111.32D sfp1A (MATa MAL2-8c SUC2 leu23,112 sfp1 $1 \Delta: \mathrm{K} 1 L E U 2)$ were used in this study. Cells were grown at $30{ }^{\circ} \mathrm{C}$ in 21 chemostats (Applikon), with a working volume of 1.51 . The medium composition was based on that described by Verduyn $e t$ al. (1992). Cultures were fed with a defined mineral medium that limited growth on a mixture of glucose and ethanol. The substrate concentrations were 200 and $50 \mathrm{mM}$ carbon (content) $(\mathrm{CmM})$ for glucose and ethanol, respectively. The addition of ethanol successfully prevented the $s f p 1 \Delta$ cultures from oscillating (Mashego et al., 2007). The dilution rate $(D)$ was set at $0.10 \mathrm{~h}^{-1}$ and the airflow at 0.5 $1 \mathrm{~min}^{-1}$. The stirrer speed was 800 r.p.m. The volume and $\mathrm{pH}$ control (pH 5) and off-gas analysis were performed as previously described 
(van Maris et al., 2003). Chemostat cultures were assumed to be in a steady-state when, after at least five volume changes, the culture dryweight, specific $\mathrm{CO}_{2}$ production rate and oxygen consumption rate changed by less than $2 \%$ during $24 \mathrm{~h}$. Steady-state samples were taken from cultures after 10 volume changes. We will refer to the moment right before the onset of the pulse as time $0(t=0)$.

Glucose anaerobic pulse. After 10 volume changes, a few seconds after the samples for the steady-state analysis were collected, the anaerobic glucose pulse experiments were started by sparging the medium reservoir and the fermenter with pure nitrogen gas (airflow $0.51 \mathrm{~min}^{-1}$, Hoek-Loos, <5 p.p.m. $\mathrm{O}_{2}$ ). Norprene tubing and butyl septa were used to minimize oxygen diffusion into the anaerobic culture. Two minutes after nitrogen sparging and just before the addition of glucose, the medium and the effluent pumps were switched off. At this time point, $60 \mathrm{ml} 5.0 \mathrm{M}$ glucose was injected aseptically through a rubber septum giving a glucose concentration of $\sim 190 \mathrm{mM}$ in the fermenter. $\mathrm{CO}_{2}$ formation was monitored online by continuously analysing the off-gas composition. Samples were collected at different time points depending on the specific analysis and the strain.

Analytical methods. Extracellular metabolite concentration and culture dry weight were determined as described previously (Cipollina et al., 2008; Postma et al., 1989). The specific consumption/ production rates for glucose and ethanol were calculated by first fitting the concentration profile of the given metabolite to a secondorder polynomial. The derivative of the polynomial was then calculated for each time point and divided by the biomass concentration measured at that time point.

In vitro measurements of enzymatic activities. The enzyme assays were performed on fresh cell-free extracts. A $60 \mathrm{ml}$ volume of culture broth was sampled from the fermenter and washed twice in ice-cold freeze buffer (10 $\mathrm{mM}$ potassium phosphate, $2 \mathrm{mM}$ EDTA, $\mathrm{pH} 7.5$ ). The pellet was resuspended in freeze buffer $(10 \mathrm{ml})$ and stored at $-20{ }^{\circ} \mathrm{C}$ in $2 \mathrm{ml}$ aliquots. Frozen samples were thawed at room temperature, washed with sonication buffer $(100 \mathrm{mM}$ potassium phosphate, $2 \mathrm{mM} \mathrm{MgCl}_{2}, \mathrm{pH} 7.5$ ) and centrifuged (5 min at $4600 \mathrm{~g}$, $4{ }^{\circ} \mathrm{C}$ ). The pellet was resuspended in $2 \mathrm{ml}$ ice-cold sonication buffer to which DTT was added to a final concentration of $1 \mathrm{mM}$. The suspension was added to $1.5 \mathrm{ml}$ Safe-Lock tubes (Eppendorf) filled beforehand with $0.75 \mathrm{~g}$ cold glass beads (Sigma). The tubes were placed in a FastPrep120 machine (Qbiogene) and shaken in four bursts of $20 \mathrm{~s}$, at speed 6 , for the efficient breakdown of the cell membranes; between the shaking cycles the tubes were cooled on ice for at least $60 \mathrm{~s}$. Unbroken cells and debris were removed by centrifugation (20 min at $47000 \mathrm{~g}, 4{ }^{\circ} \mathrm{C}$ ). The cell-free extracts obtained were used to perform the enzyme assays as described previously (Jansen et al., 2005).

Absorbance measurements were performed with a Tecan GENios Pro microtitre plate reader. All determinations were performed at $30{ }^{\circ} \mathrm{C}$ and $340 \mathrm{~nm}\left(\varepsilon_{\mathrm{NAD}(\mathrm{P}) \mathrm{H}}=6.3 \mathrm{mM}^{-1} \mathrm{~cm}^{-1}\right)$. The assays were performed in duplicate and with two different dilutions of cell extracts. Protein concentration in the cell-free extracts was measured by the Lowry method.

Microarray analysis. Sampling from chemostats, total RNA extraction, probe preparation and hybridization to Affymetrix Genechip microarrays were performed as described previously (Cipollina et al., 2008). Samples were collected at steady state and then at 5, 10, 30, 60 and $120 \mathrm{~min}$ after the pulse. The results related to steady-state samples were derived from three independent cultures; those related to the time-course analysis were derived from two independent cultures.

Acquisition and quantification of array images, global scaling and data filtering were performed using Affymetrix GeneChip Operating Software (GCOS) version 1.2, as described previously (Cipollina et al., 2008; Piper et al., 2002). To represent the variation in triplicate and duplicate measurements, the coefficient of variation (CV) was calculated as the standard deviation divided by the mean (Boer et al., 2003). For all the replicates the CV varied by less than $13 \%$, indicating a good reproducibility of the results. After global scaling (Piper et al., 2002), the expression of the housekeeping genes ACT1, HHT2 and SHR3 (encoding, respectively, actin, histone and an endoplasmic reticulum packaging chaperone) remained constant throughout the experiment, showing a CV below or around $30 \%$.

For statistical analyses of steady-state data, Microsoft Excel running the Significance Analysis of Microarrays (SAM, version 2.23) add-in was used for pair-wise comparisons between the reference and the sfp1s mutant datasets (Boer et al., 2003; Tusher et al., 2001). Enrichment for specific Munich Institute for Protein Sequences (MIPS) functional categories among the differentially expressed genes compared with their genome-wide occurrence was computed by considering a hypergeometric distribution. The obtained $P$-value indicated the statistical significance of the results.

The statistical analysis of dynamic data was performed with the Extraction of Differential Gene Expression (EDGE) software (Leek et al., 2006). We compared the time-course transcriptional profile between the $s f p 1 \Delta$ mutant and the reference strain (EDGE command: 'Identify Differentially Expressed Genes - between class'), and 1524 genes were classified as significant ( $q$ value cutoff: 0.005 ). This list of genes was then used as input for the $k$-means clustering. Each gene was represented by a vector of 12 elements: elements 1-6 represented the expression levels of the gene in the reference strain; elements 7-12 were the expression levels of the same gene in the mutant. The expression levels were normalized so that they had zero mean and unit variance. The coefficient of correlation was used as measure of the distance between vectors. The $k$-means determination was performed so as to allow the $k$ value (i.e. the number of clusters) to range from 2 up to 30 (in steps of two). A $k$ value of 12 , i.e. 12 clusters, was chosen as the one giving the best compromise between good partitioning of the dataset (the SD of each cluster was used as measure for this) and enrichment for MIPS functional categories.

To have a quantitative measure of the significance of the expression changes for a given cluster, the $Z$ score was computed for each time point using the following equation (Knijnenburg et al., 2008):

$Z=\frac{Y-n \cdot \mu}{\sqrt{n} \cdot \sigma}$

Where $Y$ is the sum of the base- 2 logarithms of the ratio between the expression at the time point under investigation and the expression at $t=0$ across all genes in the cluster $\left(t_{\mathrm{x}} / t_{0}\right), \mu$ and $\sigma$ represent the mean and SD, respectively, of the base- 2 logarithms of the ratio $t_{\mathrm{x}} / t_{0}$ across all genes in the genome, and $n$ is the number of genes in the cluster under investigation.

Quantification of RNA contents and flow cytometric analysis. Samples were collected at steady state and at the selected time points during the pulse. RNA content was chemically determined by the orcinol method (Popolo et al., 1982). The flow cytometric analysis for protein determination was performed on FITC-stained cells, as described by Vanoni et al. (1983).

\section{RESULTS}

\section{Physiology and global transcriptional profile of an sfp1 $\Delta$ mutant and the isogenic CEN.PK 113-7D strain growing under glucose/ethanol limitation}

The deletion of SFP1 in S. cerevisiae causes a slow growth phenotype during growth in shake flasks (Cipollina et al., 
2005; Jorgensen et al., 2002). To eliminate growth rate difference between CEN.PK 113-7D and its isogenic sfp1 $1 \Delta$ mutant, both strains were grown at the same growth rate of $0.10 \mathrm{~h}^{-1}$ (duplication time of $6.9 \mathrm{~h}$ ) in chemostat cultures limited by a glucose-ethanol mixture (Cipollina et al., 2008; Novick \& Szilard, 1950). As shown in Table 1, under this growth regime both strains showed highly similar physiological characteristics. The very low residual concentrations of glucose and ethanol in the broth culture indicated that both substrates were being co-consumed.

One of the most typical features of the $s f p 1 \Delta$ phenotype is a reduction in both cell size and RNA content, especially during batch growth on glucose (Cipollina et al., 2005; Jorgensen et al., 2002). The protein content, which provides a good estimate of the cell size (Alberghina \& Porro, 1993; Vanoni et al., 1983), was analysed by flow cytometry. The mutant population had a clear small size (whi) phenotype (Fig. 1, at $t=0$ ), with smaller cells both at birth and at division (Supplementary Fig. S1). The analysis of the volume distributions, performed with a Coulter counter analyser, showed results very similar to the ones obtained by the flow cytometric approach (data not shown). The total RNA content of the cells was also significantly lower in the $s f p 1 \Delta$ mutant compared with the reference strain (Fig. 2, at $t=0$ ).

The transcriptional response of yeast to the deletion of SFP1 was investigated by microarray analysis. Statistical analysis of the array data for the $s f p 1 \Delta$ and reference strains was followed by an enrichment computation of specific functional categories among the significantly differentially expressed genes (see Methods). Only the use of lenient statistical criteria [i.e. significance analysis of microarrays (SAM) analysis, false discovery rate $(\mathrm{FDR})=1 \%$, fold change $=1.5$; Supplementary Table S1] led to the identification of enriched categories. While the genes with a higher expression in the mutant were marginally enriched for the 'pheromone response, mating-type determination, sex-specific proteins' category, some of the genes displaying significant lower expression in the mutant could be more specifically related to ribosome biogenesis ( $R P A 14, R P B 5$, $R P A 190, R P A 135$ and RRN3). These genes encoded subunits of the RNA polymerase I, the enzymic complex responsible for the transcription of rRNA (Moss, 2004). Since rRNA represents $\sim 80 \%$ of the total cellular RNA (Warner, 1999), this finding was consistent with the lower RNA content typical of $s f p 1 \Delta$ mutant cells (Fig. 2).

\section{Adaptation of S. cerevisiae CEN.PK 113-7D and sfp1 $1 \Delta$ strains to fast metabolic rates}

To investigate the response of the $\operatorname{sfp} 1 \Delta$ mutant to a sudden increase in the external glucose concentration, the carbon-limited chemostat cultures of both mutant and reference strains were pulsed with a highly concentrated glucose solution (final concentration $\sim 190 \mathrm{mM}$ ). Since the sfp1 $1 \Delta$ phenotype is especially evident during anaerobic growth and hence under fully fermentative growth
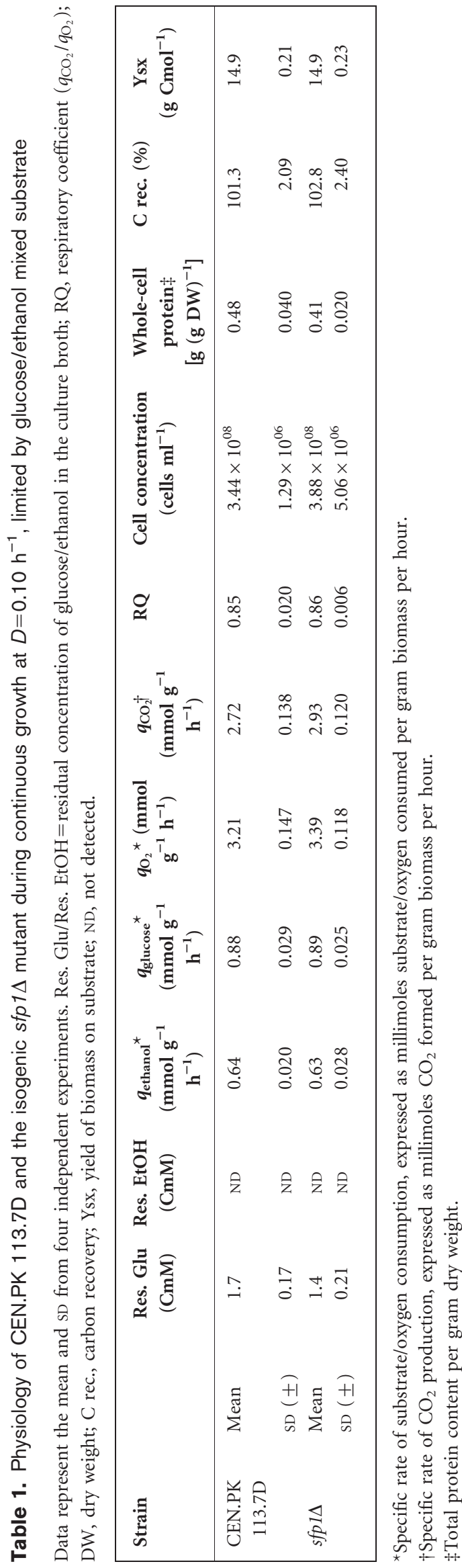


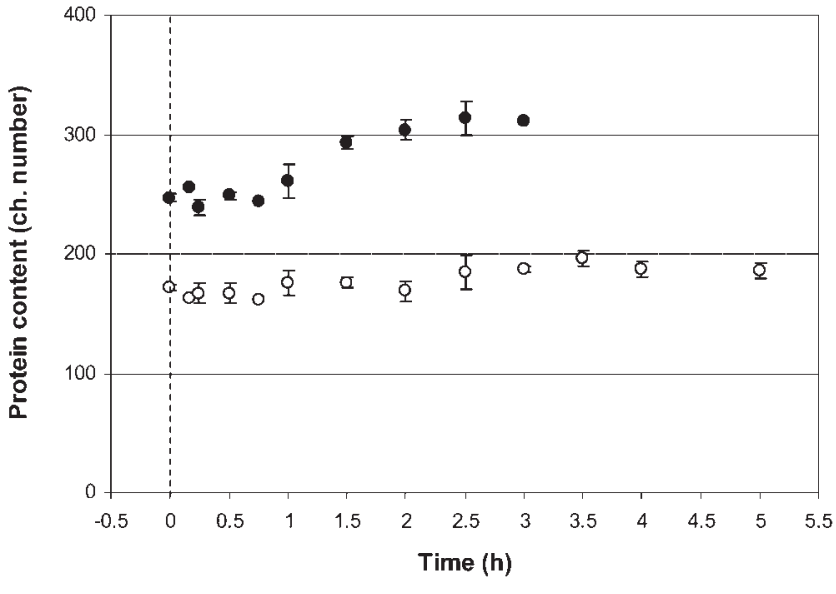

Fig. 1. Protein content per cell at steady state ( $t=0$, dashed line) and after the glucose pulse. Samples were collected at the indicated time points. The average protein content per cell [expressed as channel (ch.) number, relative units] was determined from the protein content distribution obtained by flow cytometry on FITC-stained cells. Data are shown for the reference $(0)$ and the sfp1 $1 \Delta$ mutant strain $(\bigcirc)$. The average and the mean deviation of two independent experiments are reported.

conditions (Cipollina et al., 2005, 2008), we decided to switch the cultures to anaerobiosis at the time of the glucose pulse. Moreover, we have recently reported an extensive analysis of the response of $S$. cerevisiae CEN.PK 113-7D to an anaerobic glucose pulse (van den Brink et al.,

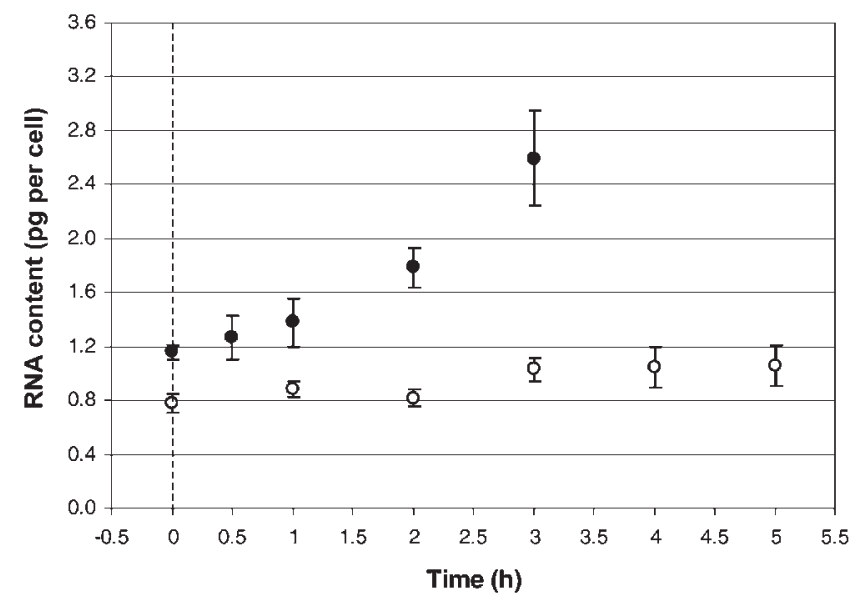

Fig. 2. Changes in the RNA content per cell in response to the glucose pulse. Samples were collected at steady state $(t=0$, dashed line) and at the indicated time points after the pulse. The total RNA content was measured with the orcinol method. Data are reported for the reference $(\bullet)$ and the $\operatorname{sfp} 1 \Delta$ mutant strain $(\bigcirc)$. The average and the mean deviation of two independent experiments are reported.
2008). Here, by choosing a similar experimental set-up, we could specifically focus on the phenotypic traits due to SFP1 deletion, leaving the detailed description of the behaviour of the reference strains to the previous manuscript.

After glucose addition, both strains, which displayed a fully respiratory metabolism in carbon-limited cultures, immediately switched to fermentative metabolism, as revealed by ethanol production (Fig. 3a). Similarly, the specific glucose consumption rate $\left(q_{\text {glucose }}\right)$ immediately increased for both strains (Fig. 3b). After the initial rapid induction, $q_{\text {glucose }}$ increased more slowly until it reached a plateau $\sim 2.5 \mathrm{~h}$ after the glucose pulse. The magnitude of $q_{\text {glucose }}$ throughout the pulse was substantially lower for the sfp $1 \Delta$ mutant than for the reference strain (about twofold lower after $2.5 \mathrm{~h}$ ). This resulted in a longer fermentation time for the mutant (Supplementary Fig. S2).

(a)

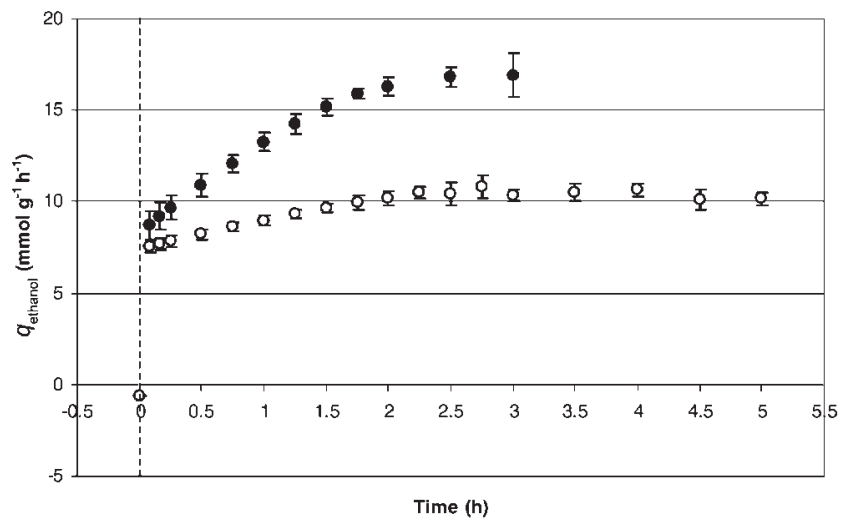

(b)

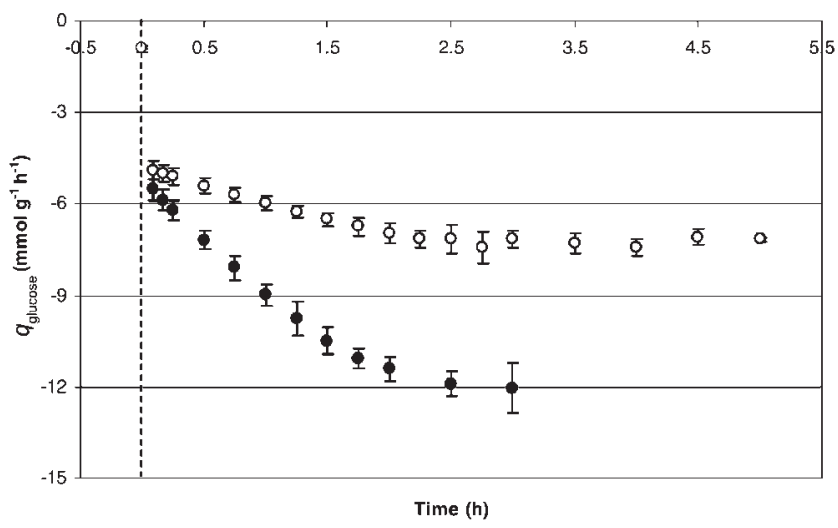

Fig. 3. Time-course profile of ethanol specific production rate (a) and glucose specific consumption rate (b) at steady state and after the glucose pulse ( $t=0$, dotted line). The $q$ values are reported for the time window in which the glucose concentration remained above $50 \mathrm{mM}$ (i.e. $3 \mathrm{~h}$ for the reference strain and $5 \mathrm{~h}$ for the mutant; see Supplementary Fig. S2). Data are reported for the reference $(\bullet)$ and the $\operatorname{sfp} 1 \Delta$ mutant strain $(\bigcirc)$. Data represent the average and the mean deviation of four independent experiments. 
Although $s f p 1 \Delta$ could increase its ethanol production rate, and therefore its glycolytic flux, the deletion of SFP1 clearly resulted in a strain with lower maximum glycolytic rates compared with the reference strain. One likely explanation of the inability of $\mathrm{sfp} 1 \Delta$ to channel high carbon fluxes could be a lower glucose-uptake capacity. In yeast, the hexose transporters are mainly encoded by seven genes (HXT1-HXT7), whose expression is mostly (although not exclusively) regulated at the transcriptional level (Ozcan et al., 1996). The transcriptional response of HXT genes in the $s f p 1 \Delta$ mutant was very similar to that of the reference strain, apart from a slightly lower induction of HXT3 and HXT4 (data not shown), suggesting that glucose uptake was not responsible for the slower glycolytic flux in the sfp $1 \Delta$ mutant. Alternatively, this lower glycolytic flux could result from a lower maximum capacity $\left(V_{\max }\right)$ of the glycolytic enzymes. This $V_{\max }$ can be quantified in vitro by assaying the specific activity of the glycolytic enzymes, which is expressed as enzyme units per milligram of protein. The $V_{\max }$ of most glycolytic enzymes increased between 30 and $180 \mathrm{~min}$ following glucose addition (Supplementary Fig. S3), and both their profiles and the intensity of the change were very similar for both strains. The changes in the transcript levels of these enzymes were unaffected in the mutant strain, and mirrored rather well the changes of the activity profiles (data not shown).

Conversely, the capacities of the first and the last enzymes of the glycolytic pathway, i.e. hexokinase (Hxk) and alcohol dehydrogenase (Adh), were altered in the mutant phenotype compared with the reference strain (Fig. 4). The specific activity of Hxk at steady state and throughout the pulse experiment was lower $(\sim 1.5$-fold $)$ in the $s f p 1 \Delta$ strain. While $H X K 1$ and GLK1 showed similar expression levels and regulation in response to glucose, HXK2 (encoding the predominant hexokinase isoform during growth on glucose; Johnston, 1999) showed, in good agreement with Hxk $V_{\max }$, a steady twofold lower expression in the mutant strain (Fig. 4). This suggested that the difference in Hxk capacity between the reference and mutant strains was predominantly regulated at the transcriptional level.

The activity profile for Adh was similar to that for Hxk, with lower values in the mutant strain $(\sim 1.7$-fold) throughout the experiment. The correlation between activity and transcript levels for this enzyme was not straightforward, since in yeast six genes encode Adh. Fig. 4 shows that while $A D H 2$ was similarly downregulated in both strains after glucose addition, the regulation of the remaining $A D H$ genes was affected in the mutant strain. This might correlate with the observed differences in enzyme activity.

Finally, enolase (Eno) activity, although increasing after glucose addition for both strains, was slightly lower in the sfp $1 \Delta$ mutant after the pulse. Such a difference could not be predicted from the mRNA levels (Fig. 4) and suggested a post-transcriptional regulation of the enzyme capacity (i.e. by protein synthesis and turnover or post-translational modification).

\section{Effect of SFP1 deletion on the global transcriptional response to the glucose pulse}

We have recently investigated the general transcriptional response of budding yeast to an anaerobic glucose pulse (van den Brink et al., 2008). Here we specifically studied the effect of SFP1 deletion on the transcriptional reprogramming triggered by this perturbation. Statistical analysis revealed that the expression profiles of 1524 genes differed significantly between the reference and the $s f p 1 \Delta$ strain after the anaerobic glucose pulse (Supplementary Table S2). These 1524 genes were spread across twelve clusters according to their expression profiles (Supplementary Fig. S4). Each cluster was subsequently searched for enrichment in specific MIPS functional categories and promoter elements.

As reported in Table 2, significant enrichment was found for most of the 12 clusters. However, the biological meaning of such enrichment was in many cases unclear. For example, clusters 5, 9, 11 and 12 were enriched for categories related to carbon metabolism and cell cycle progression, and cluster 6 comprised a significant number of genes involved in the stress response. However, the number of genes represented in each cluster, although statistically significant, was too low compared with the total number of genes in the category to allow meaningful biological conclusions to be drawn. Neither clear patterns nor relationships were found between these genes and the observed phenotype, and no striking differences in expression profiles between the two strains were observed for these genes. Clusters 1 and 7 showed no enrichment for MIPS categories, while cluster 3 showed some enrichment for a small category related to vitamins/cofactors transport. Finally, clusters 2, 4, 8 and 10 showed the strongest differences in the expression profiles between the two strains and the most significant enrichments for functional categories.

Genes belonging to cluster 2 showed a comparable initial induction in the two strains until $30 \mathrm{~min}$, but then diverged in the two strains during the remaining fermentation (Fig. 5). This cluster was enriched for genes involved in amino acid metabolism and translation elongation, and for ribosomal protein genes, and accordingly promoter elements recognized by the transcription factors Fhl1 and Rap1 were over-represented (Table 2; Knijnenburg et al., 2007). Genes grouped in clusters 4 and 10 showed a delay in induction in the $\operatorname{sfp} 1 \Delta$ strain as compared with the reference strain. These clusters comprised genes involved in ribosome biogenesis, translation initiation and nucleotide metabolism, and showed accordingly a significant enrichment for RRPE and PAC promoter elements (Table 2) (Hughes et al., 2000).

Finally, cluster 8 displayed the opposite trend between the reference and the $s f p 1 \Delta$ strain, and was enriched for genes involved in proteasomal degradation. Thirteen out of 106 genes in this cluster encoded either structural or regulatory subunits of the $26 \mathrm{~S}$ proteasome involved in the degradation of ubiquitinated substrates. The binding site recognized by 
(a)
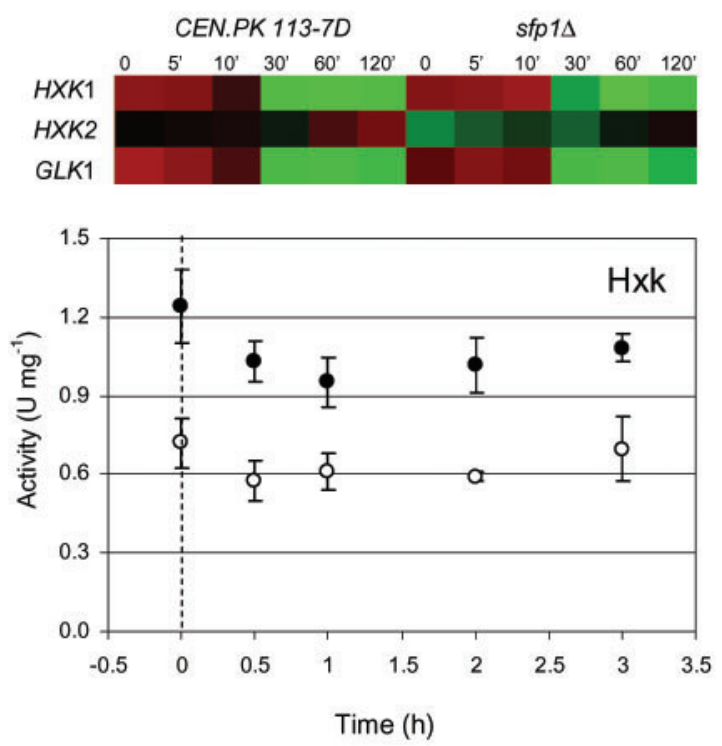

(c)
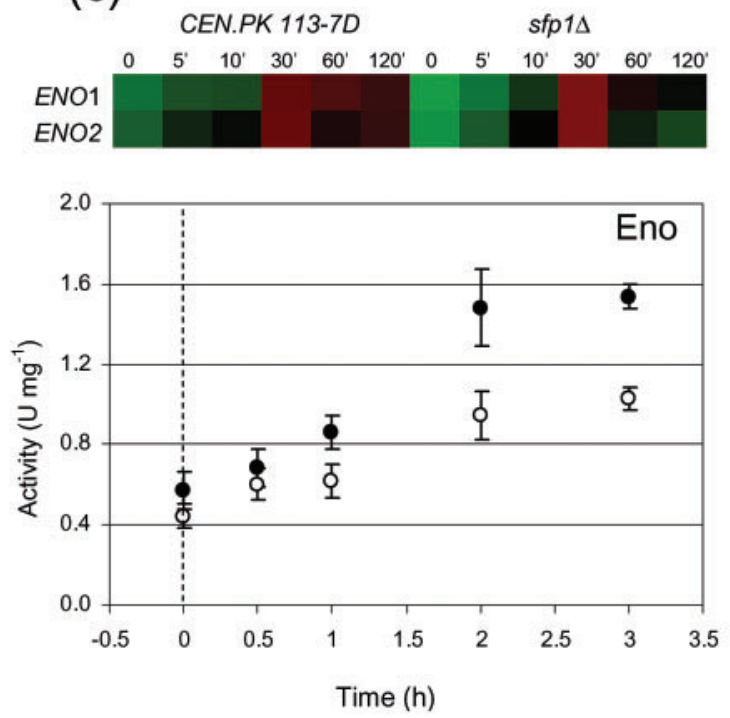

(b)
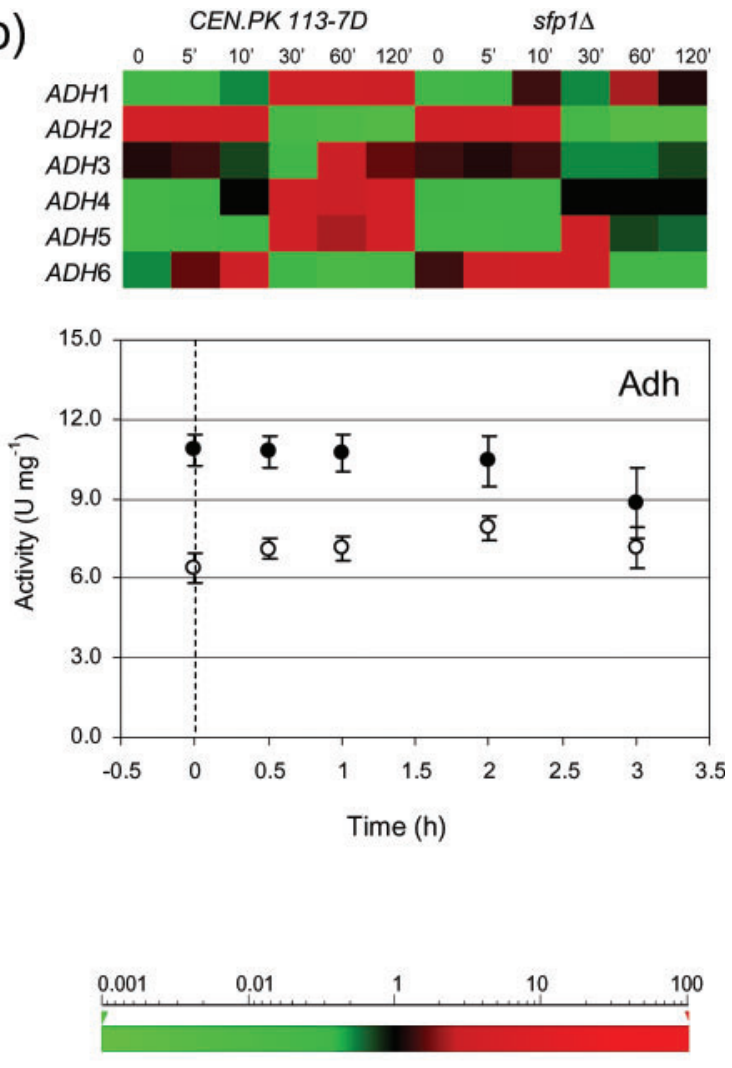

Fig. 4. Transcript levels and specific activity of hexokinase (a), alcohol dehydrogenase (b) and enolase (c) at steady state ( $t=0$, dotted line) and after the glucose pulse. Transcript data are reported in the heat maps and derive from samples collected at steady state and at 5, 10,30,60 and $120 \mathrm{~min}$ after glucose addition. The transcript levels were normalized by dividing the expression level of each gene at a given time point by the mean expression level over the 12 conditions (six time points, two strains). The key to the heat maps is shown to the right of panel (c). Samples for enzyme activity determination were collected at steady state and 30,60,120 and $180 \mathrm{~min}$ after the addition of glucose. Data are reported for the reference $(\bullet)$ and the sfp1 $\Delta$ strain $(\bigcirc)$, and represent the average and the mean deviation of two independent experiments.

Rpn4, a transcription factor specific for proteasome genes (Xie \& Varshavsky, 2001), was accordingly enriched in cluster 8 (Table 2). The proteasome $26 \mathrm{~S}$ is involved, among other functions, in the post-transcriptional regulation of many important factors required for proper control of cell cycle progression (DeSalle \& Pagano, 2001; Futcher, 1996). The altered regulation of these genes in the $s f p 1 \Delta$ strain might therefore be related to its small size phenotype. However, in the present work, this hypothesis was not investigated further. 
Table 2. Enrichment for MIPS functional categories and known transcription factor (TF) binding sites

Horizontal lines within the body of the table demarcate the MIPS category combinations that add up to the number of genes in each cluster.

\begin{tabular}{|c|c|c|c|c|c|c|c|}
\hline \multirow[t]{2}{*}{ Cluster } & \multirow{2}{*}{$\begin{array}{l}\text { Number of genes in } \\
\text { the cluster }\end{array}$} & \multicolumn{3}{|c|}{ Enrichment for MIPS category ${ }^{*}$} & \multicolumn{3}{|c|}{ Enrichment for TF binding site ${ }^{\star}$} \\
\hline & & MIPS category $\dagger$ & $\begin{array}{c}\text { Number of genes in } \\
\text { the MIPS } \\
\text { category }\end{array}$ & $P$ value & TF binding site $\dagger$ & $\begin{array}{l}\text { Number of genes with } \\
\text { the TF binding site }\end{array}$ & $P$ value \\
\hline 1 & 90 & No MIPS categories over-represented & - & - & Hsflp (TTCynnnnnnTTC) (13) & 133 & $3.2 \times 10^{-08}$ \\
\hline \multirow[t]{5}{*}{2} & 134 & 12.01.01. ribosomal proteins $(47)$ & 227 & $1.7 \times 10^{-35}$ & Bas1 (TGACTC) (9) & 36 & $3.5 \times 10^{-08}$ \\
\hline & & 01.01. amino acid metabolism $¥(21)$ & 239 & $1.8 \times 10^{-08}$ & Fhl1 (TGTayGGrtg) (47) & 203 & $6.5 \times 10^{-38}$ \\
\hline & & 01.03.01. purine nucleotide metabolism (9) & 63 & $5.5 \times 10^{-06}$ & Gcn4 (TGAsTCa) (14) & 192 & $4.4 \times 10^{-05}$ \\
\hline & & 12.04.02. translation elongation (5) & 19 & $3.5 \times 10^{-05}$ & Rap1 (cayCCrtrCa) (30) & 157 & $2.8 \times 10^{-21}$ \\
\hline & & & & & Sfp1 (ayCcrtAcay) (16) & 51 & $2.2 \times 10^{-15}$ \\
\hline 3 & 77 & 20.01.25. vitamin/cofactor transport (4) & 14 & $1.8 \times 10^{-05}$ & - & - & - \\
\hline \multirow[t]{4}{*}{4} & 168 & 12.04.01. translation initiation (16) & 39 & $5.8 \times 10^{-16}$ & RRPE (GAAAWWTT) (101) & 1100 & $<1.0 \times 10^{-20}$ \\
\hline & & 12.10. aminoacyl-tRNA-synthetases (12) & 39 & $1.6 \times 10^{-10}$ & & & \\
\hline & & 12.01. ribosome biogenesis $(22)$ & 290 & $6.1 \times 10^{-06}$ & Fhll (TGTayGGrtg) (20) & 203 & $2.8 \times 10^{-07}$ \\
\hline & & 11.02.01. rRNA synthesis (8) & 55 & $8.3 \times 10^{-05}$ & & & \\
\hline \multirow[t]{3}{*}{5} & 145 & 01.06. lipid, fatty acid and isoprenoid metabolism (22) & 281 & $2.8 \times 10^{-07}$ & Hap1 (GGnnaTAnCGs) (10) & 73 & $4.9 \times 10^{-06}$ \\
\hline & & 02. energy (24) & 353 & $1.0 \times 10^{-06}$ & & & \\
\hline & & $\begin{array}{l}\text { 01.05. C-compound and carbohydrate metabolism } \\
\text { (26) }\end{array}$ & 495 & $4.0 \times 10^{-05}$ & & & \\
\hline \multirow[t]{2}{*}{6} & 68 & 32.01. stress response $(20)$ & 437 & $1.3 \times 10^{-08}$ & Hsf1 (TTCynnnnnnTTC) (19) & 133 & $4.4 \times 10^{-17}$ \\
\hline & & & & & Msn2 (mAGGGGsgg) (7) & 65 & $4.8 \times 10^{-06}$ \\
\hline \multirow[t]{2}{*}{7} & 161 & No MIPS categories over-represented & - & - & Dig1 (TgAAAca) (13) & 144 & $6.2 \times 10^{-05}$ \\
\hline & & & & & Ste12 (tgAAAC) (18) & 231 & $1.7 \times 10^{-05}$ \\
\hline 8 & 106 & 14.13.01.01. proteasomal degradation (13) & 127 & $1.4 \times 10^{-07}$ & Rpn4 (GGTGGCAAA) (10) & 93 & $2.7 \times 10^{-06}$ \\
\hline 9 & 111 & 01.06.01.07. isoprenoid biosynthesis (6) & 39 & $4.9 \times 10^{-05}$ & Mbp1 (ACGCGT) (17) & 165 & $2.5 \times 10^{-09}$ \\
\hline \multirow[t]{4}{*}{10} & 257 & 11.04.01. rRNA processing $(96)$ & 168 & $2.0 \times 10^{-95}$ & RRPE (GAAAWWTT) (181) & 1100 & $<1.0 \times 10^{-20}$ \\
\hline & & & & & PAC (GMRATGARnT) (126) & 538 & $<1.0 \times 10^{-20}$ \\
\hline & & 11.02.01. rRNA synthesis (15) & 55 & $2.2 \times 10^{-09}$ & & & \\
\hline & & 01.03. nucleotide metabolism (25) & 222 & $2.5 \times 10^{-06}$ & & & \\
\hline 11 & 76 & 2.10. tricarboxylic acid pathway (5) & 31 & $2.8 \times 10^{-05}$ & Skn7 (GnCnnGsCs) (11) & 156 & $1.9 \times 10^{-06}$ \\
\hline 12 & 131 & 10. cell cycle and DNA processing (40) & 975 & $6.4 \times 10^{-06}$ & - & - & - \\
\hline
\end{tabular}

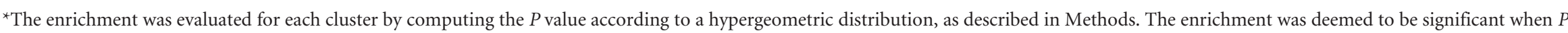
$<1.0 \times 10^{-04}$. Each cluster contained a proportion of genes of unknown function ranging from 10 to $30 \%$.

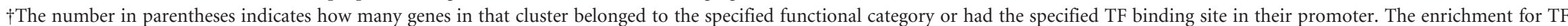

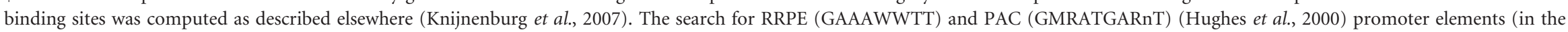
region from -800 to -1 ) was performed by using the software RSATools (van Helden et al., 2000).

$\ddagger$ In particular, lysine, cysteine and glycine metabolism. 

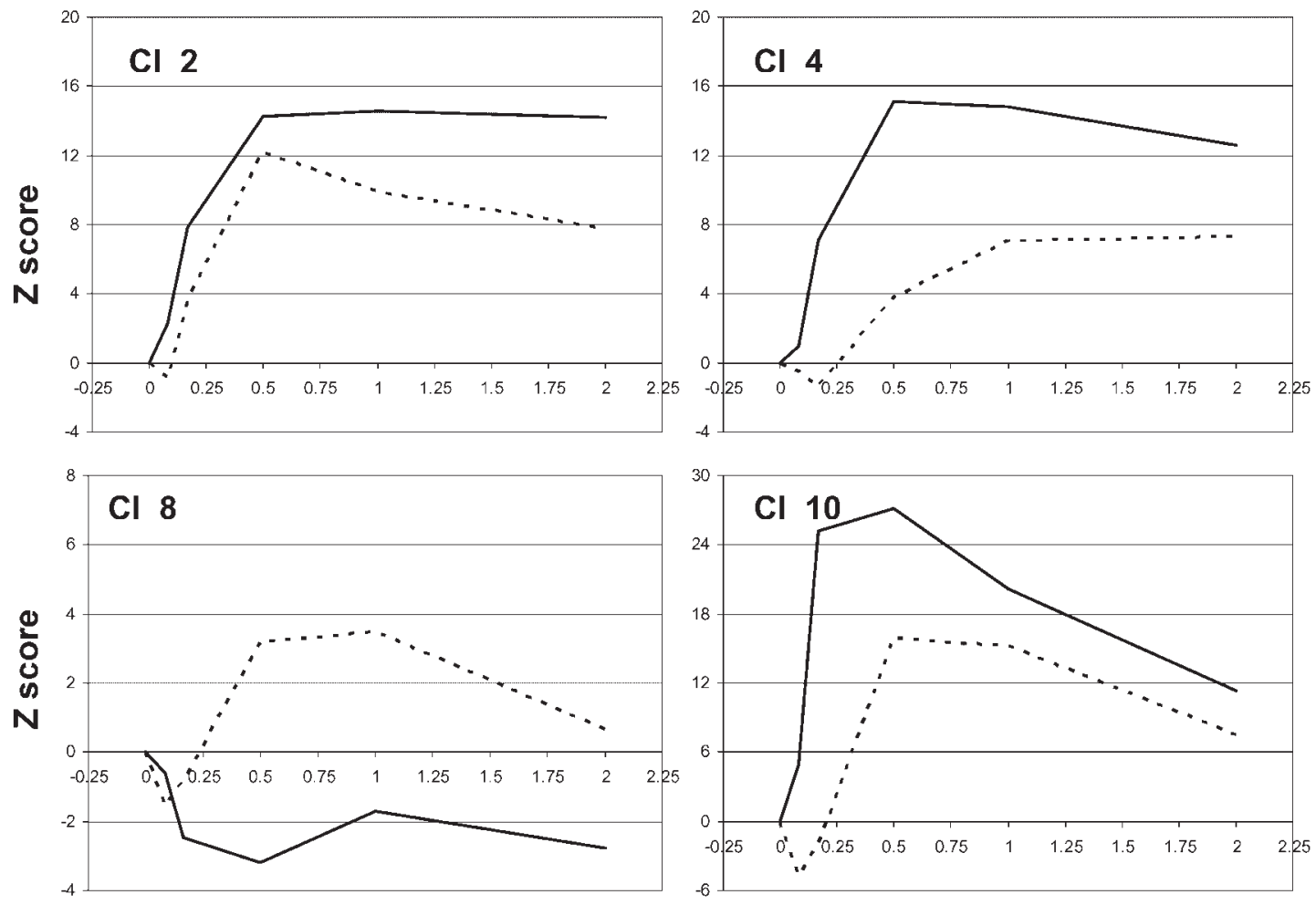

Time (h)

Fig. 5. $Z$ score profiles of clusters $(\mathrm{Cl}) 2,4,8$ and 10 for the reference (solid line) and the sfp $1 \Delta$ (dashed line) strain. The $Z$ score indicates both the significance of the change at time $t_{x}$ relative to $t=0$ and the direction of the change (up- $/$ downregulation). It was calculated as described in Methods.

\section{Effect of SFP1 deletion on RiBi and RP gene expression in response to glucose pulse}

We have previously shown that the deletion of SFP1 in $S$. cerevisiae resulted in decreased expression of the genes involved in ribosome biogenesis (the so called 'RiBi cluster'), but did not affect expression of RP genes (Cipollina et al., 2008). These results suggested that Sfp1 was not involved in the transcriptional regulation of RP genes when S. cerevisiae was grown at a slow growth rate under limited carbon supply. Here, the addition of a glucose excess to carbon-limited cultures allowed us to analyse the involvement of Sfp1 in RiBi and RP gene transcriptional modulation when the cells express their full potential for sugar utilization and growth.

In agreement with our recently published data (van den Brink et al., 2008), RiBi (clusters 4 and 10) and RP (cluster 2) genes responded with different dynamics to sudden glucose excess (Fig. 6). We focused on the 96 rRNA processing genes belonging to cluster 10 and the $47 \mathrm{RP}$ genes belonging to cluster 2 . While the $47 \mathrm{RP}$ genes were similarly induced in both strains in the 30 min following glucose addition (Fig. 6a), the induction of the RiBi genes showed a substantial delay in the mutant strain and remained weaker throughout the whole fermentation (Fig. 6b). This finding indicated that while SFP1 was not essential for the early induction of RP genes, it was required for the initial transcriptional burst of the RiBi genes. After $30 \mathrm{~min}$, RP genes were downregulated in the mutant strain in accordance with previous reports showing that during shake-flask growth the expression of these genes is reduced in a $\operatorname{sp} 1 \Delta$ strain. Interestingly, genes encoding factors required for nucleotide metabolism and translation initiation showed a response similar to the RiBi genes, while genes encoding factors required for amino acid biosynthesis and translation elongation showed a response similar to that of the RP genes (Table 2).

The observed defect in the induction of RiBi genes was consistent with the smaller increase in the total RNA content in the $\operatorname{sfp} 1 \Delta$ mutant compared with the reference strain (Fig. 2).

\section{Modulation of cell size and cell cycle progression}

One of the typical traits of an $s f p 1 \Delta$ mutant is the small size (also called whi) phenotype (Cipollina et al., 2005; 
(a)

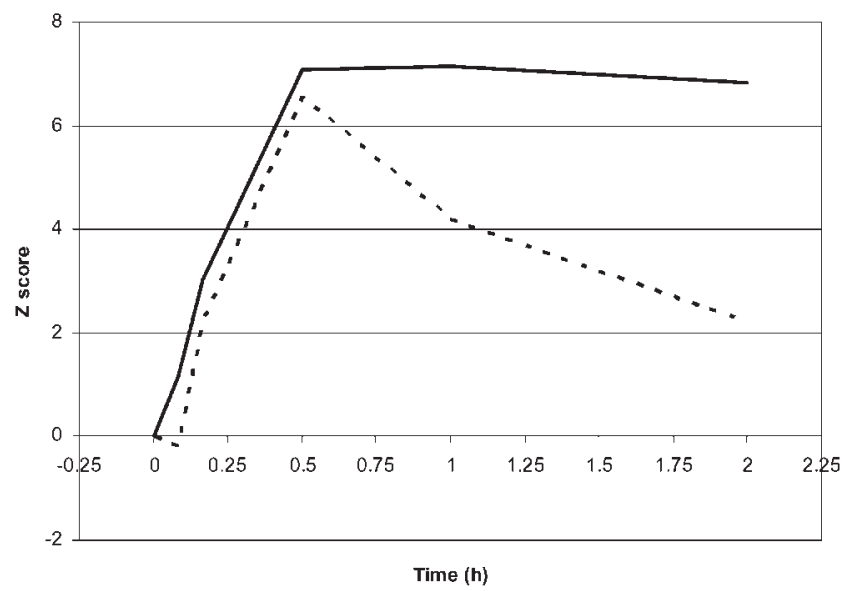

(b)

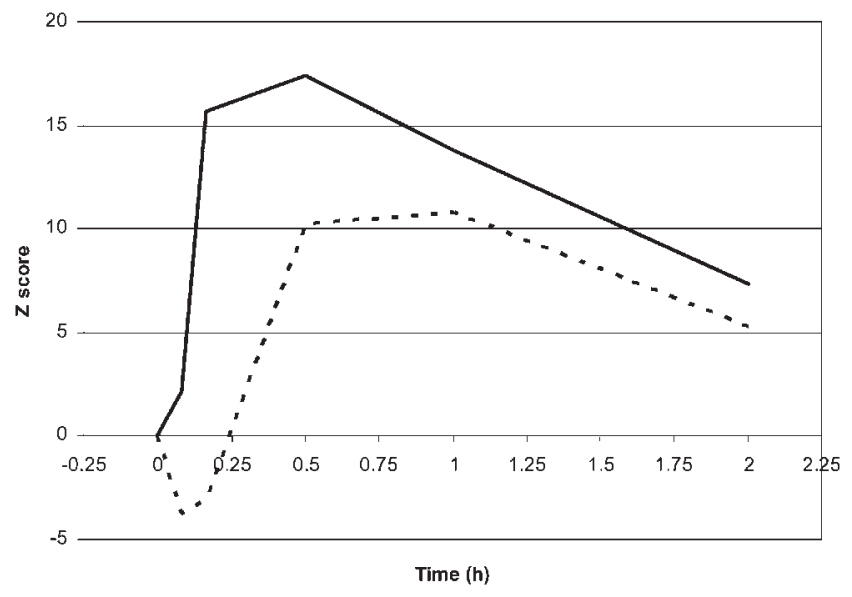

Fig. 6. $Z$ score profiles of RiBi and RP genes in response to the glucose pulse. The $Z$ score profiles relating to the $47 \mathrm{RP}$ genes from cluster 2 (a) and the 96 'rRNA processing' genes belonging to cluster 10 (b) are reported for the reference (solid line) and the $\operatorname{sfp} 1 \Delta$ (dashed line) strain. The $Z$ score indicates both the significance of the change at time $t_{x}$ relative to $t=0$ and the direction of the change (up-/downregulation). It was calculated as described in Methods.

Jorgensen et al., 2002). Accordingly, during aerobic ethanol/ glucose-limited growth, the sfp $1 \Delta$ strain showed a whi phenotype (Fig. 1). Approximately $1 \mathrm{~h}$ after the glucose pulse, the cell size of the reference strain increased to reach a value $30 \%$ higher than the steady-state value at the end of the fermentation. Conversely, the size of the mutant cells remained steady for about $2 \mathrm{~h}$ after the glucose pulse, then finally slowly increased to reach a value as little as $10 \%$ higher than the steady-state size (Fig. 1). This resulted in an increase in the difference in cell size between the two strains caused by the addition of glucose excess, consistent with previous reports (Cipollina et al., 2005).

It has been shown elsewhere that after the addition of excess glucose to non-repressed cells, a yeast culture starts to grow (i.e. produce biomass) at its maximum specific growth rate $\left(\mu_{\max }\right)$ (Alberghina et al., 1998). Another typical effect induced by a glucose pulse is the increase in cell size, which is attained through a tight coordination between growth and cell cycle progression (Alberghina et al., 1998; Flick et al., 1998). During anaerobic batch growth on glucose, the reference and mutant strains grew with $\mu_{\max }$ values of 0.13 and $0.34 \mathrm{~h}^{-1}$, respectively. After the pulse, we observed a similar behaviour for both strains, with a transient decrease of the budding index (BI) from $\sim 40$ to $30 \%$ ( $1 \mathrm{~h}$ after the pulse) followed by an increase to about $60 \%$ (Supplementary Fig. S5a). This could be explained by a temporary delay at the G1 to $S$ transition (Alberghina et al., 1998; Flick et al., 1998). Accordingly, during the first $2 \mathrm{~h}$ after the pulse, no significant increase in cell number was observed for both strains, indicating that the cellular proliferation had temporarily slowed down (Supplementary Fig. S5b). The combined effect of this delay in the G1 to $S$ transition and the change in the specific growth rate resulted in the observed adjustment to the new size setting, which was different for the two strains (Fig. 1).

\section{DISCUSSION}

\section{The involvement of Sfp1 in cell size modulation and transcriptional control is differentially modulated by the growth conditions}

The use of chemostat cultures has recently been proven to be a very good approach to study the role of Sfp1 in cellsize modulation and transcriptional control (Cipollina et al., 2008). It allowed us to grow both a mutant deleted for SFP1 and its reference isogenic strain at the same growth rate under different carbon limitations. This would not have been possible in shake-flask cultures due to the slow growth phenotype of the $s f p 1 \Delta$ mutant (Cipollina et al., 2005; Jorgensen et al., 2002).

The present work concludes a series of chemostat experiments performed to assess the influence of the carbon source on $s f p 1 \Delta$ phenotype, independent of the growth rate-related phenotype. Here, we analysed the physiology of cultures growing aerobically under glucose/ethanol limitation. This growth regime created a metabolic condition intermediate between the previously analysed conditions (Cipollina et al., 2008). During anaerobic glucose-limited growth, the substrate was completely fermented to ethanol and the glycolytic flux was high $\left[q_{\text {glucose }}\right.$ approx. $-3.2 \mathrm{mmol}$ (g biomass) ${ }^{-1} \mathrm{~h}^{-1}$ ] (Cipollina et al., 2008; Daran-Lapujade et al., 2007). During ethanol-limited growth, the cultures showed a purely respiratory metabolism and the glycolytic flux proceeded backwards (gluconeogenesis) for biosynthetic purposes (de Jong-Gubbels et al., 1995). In the present study, during aerobic glucose/ethanol limitation, with glucose supplying $80 \%$ of the substrate carbon, metabolism was purely respiratory and glycolysis fully active, although the rate of glucose consumption was lower compared with 
the anaerobic growth previously analysed $\left[q_{\text {glucose }}\right.$ approx. $-0.90 \mathrm{mmol}$ (g biomass) $\left.{ }^{-1} \mathrm{~h}^{-1}\right]$. The finding that the $\operatorname{sp} 1 \Delta$ mutant showed a clear whi phenotype in all the growth regimes so far analysed indicates that the involvement of SFP1 in the modulation of cell size is independent of the growth rate, the supplied carbon source and the metabolism of the cell.

Conversely, the involvement of Sfp1 in RiBi transcriptional regulation appears to be strongly related to the carbon source provided and the growth regime. We observed that when no glucose was present in the medium (ethanollimited growth), the transcriptional profile of an $s f p 1 \Delta$ mutant was similar to that of the reference strain. During anaerobic glucose-limited growth, serious transcriptional alterations involving the RiBi cluster and the RNA polymerase I coding genes were observed for the mutant strain (Cipollina et al., 2008). Here, under the intermediate metabolic regime typical of glucose/ethanol-limited growth, some transcriptional alteration was present, related to the small but important cluster of RNA Pol I coding genes. These findings suggest that a sustained glycolytic flux and/or a fermentative metabolism might generate a signal stimulating the Sfp 1 transcriptional role. It has been reported previously that the presence of an active glycolytic flux contributes to the activation of important nutrientsignalling pathways that regulate growth and metabolism (Li et al., 2006; Newcomb et al., 2003; Rolland et al., 2001, 2002). Sfp1 might be involved in this regulatory network.

\section{Sfp1 is required for efficient induction of $\mathrm{RiBi}$, but not RP, genes in response to glucose excess}

The involvement of Sfp1 in transcriptional regulation of $\mathrm{RiBi}$ and RP genes has so far been a controversial issue. Most of the data derived from shake-flask cultures indicate that during growth on glucose, Sfp1 is involved in transcriptional regulation of both RiBi and $\mathrm{RP}$ genes (Jorgensen et al., 2004; Marion et al., 2004). The former gene cluster is characterized by the presence of RRPE and PAC elements in the promoter regions. The latter is particularly enriched for the promoter elements recognized by the transcription factors Rap 1 and Fhl1. All attempts to prove a physical interaction between Sfpl and the RiBi gene promoters have failed, while some weak interactions have been observed between Sfp1 and the promoters of some RP genes (Fingerman et al., 2003; Harbison et al., 2004; Jorgensen et al., 2004; Marion et al., 2004). Recently it has been shown that the protein Stb3 binds the RRPE elements in vivo and that it is required for the proper regulation of RRPE-containing genes in response to glucose. It has been suggested that Sfp1 acts upstream of Stb3 in modulating the transcription of RiBi genes (Liko et al., 2007).

We recently proposed that the main target of Sfp 1 is the $\mathrm{RiBi}$ cluster, and that the downregulation of RP genes observed in the $\operatorname{sflp} \Delta$ mutant during shake-flask growth might be a secondary effect due to the slow growth rate of the mutant strain. The glucose-pulse experiment presented here was successful in further supporting this hypothesis. Here we conclusively show that Sfp1 is required for the efficient and rapid increase in expression of RiBi genes in response to the glucose pulse, but not for the early transcriptional induction of RP genes (Fig. 6). Since the transcriptional regulation of RP and RiBi genes has been shown to be tightly co-ordinated (Griffioen et al., 1996; Laferte et al., 2006), we suggest that the downregulation of $\mathrm{RP}$ genes observed in the mutant strain from $30 \mathrm{~min}$ after glucose addition is due to the adjustment of RP expression levels to the low expression of RiBi and RNA Pol I genes (Fig. 6). This would in turn result in the slow growth-rate phenotype typical of $s f p 1 \Delta$ cells.

The observation that RiBi genes are still able to respond to glucose addition in the $\operatorname{sp} 1 \Delta$ strain, although with some delay and to a lesser extent compared with the reference strain, indicates that Sfp1 is not the only regulator of these genes. In the absence of SFP1, alternative mechanisms appear to operate to modulate the expression of RiBi genes depending on nutrient availability. Finally, while the observation of a weak binding of Sfp1 to RP promoter genes might suggest a role in the regulation of these genes (Fingerman et al., 2003; Jorgensen et al., 2004; Marion et al., 2004), our data indicate that this is not a primary function of Sfp1.

\section{Sfp1 might play a role at the crossroads of glycolysis regulation and ribosome biogenesis}

Most of the information so far available indicates that the phenotype of an sfp $1 \Delta$ mutant is much more evident during fermentative growth on glucose than during respiratory growth on a non-fermentable carbon source (ethanol or glycerol). In the present work we chose an experimental set-up that allowed us to analyse the transition from a condition of respiratory growth in which the mutant phenotype was only partly present to a condition of anaerobic batch growth on glucose (fully fermentative). Therefore, we expected the mutant phenotype to dominate during the time interval that followed the perturbation. In particular, since at steady state both strains were growing at the same specific growth rate, the observed alterations in the early response to glucose addition could be ascribed to the absence of SFP1 rather than to growth rate-related defects.

Besides a different transcriptional phenotype, we observed significant defects in the induction of the glucose-specific consumption rate in the $\operatorname{sfp} 1 \Delta$ mutant within minutes of glucose addition. This clearly indicated that deletion of SFP1 has a negative effect on the glycolytic activity of the cell. As reported in Results, the $V_{\max }$ of most glycolytic enzymes at steady state and in response to glucose showed no difference between the two strains. The reported enzymic activities were normalized based on the protein content of the cell cultures used for the assays (see Methods). Since the $\operatorname{sfp} 1 \Delta$ strain had a lower protein 
content than the reference strain at steady state and throughout the pulse experiment (Fig. 1, Table 1), the absolute capacity of glycolytic enzymes in the mutant cultures was lower than that of the reference strain. The effect of such lower activities was not evident at steady state, when the glucose consumption rate was relatively low (Table 1). However, after the addition of excess glucose the cells were forced to exploit their maximum glycolytic potential. Under this condition the lower glycolytic potential of the $s f p 1 \Delta$ cultures could partly explain the observed reduction in the glycolytic flux. Nevertheless, it is noteworthy that the deletion of SFP1 did not affect the induction (relative to $t=0$ ) and thus the regulation of most glycolytic enzymes in response to glucose addition (Supplementary Fig. S3).

Conversely, the enzyme hexokinase showed differential expression between the two strains, with both lower transcriptional levels and lower activity in the sfp $1 \Delta$ mutant throughout the experiment. This enzyme plays a key role in the regulation of central carbon metabolism for two reasons (Westergaard et al., 2007). It catalyses the first step of glycolysis, i.e. the phosphorylation of glucose, and it is involved in the regulation of protein kinase A and Mig1, which control growth and central metabolism in response to nutrient availability and environmental conditions (Ahuatzi et al., 2007; Moreno et al., 2005; Rolland et al., 2001). Given the crucial role of Hxk in the regulation of glycolytic flux, the reduced $V_{\max }$ observed for this enzyme could be related to the lower glycolytic rate of the mutant strain. In addition, the observed defects in Hxk2 expression and activity may be linked to the carbon source-dependent phenotype of the $s f p 1 \Delta$ mutant. Whether Sfp1 affects Hxk activity via a direct mechanism or lower Hxk activity is caused by the low RNA or protein content typical of the mutant strain remains to be elucidated.

The finding that the deletion of SFP1 simultaneously affects the induction of both RiBi gene transcription and glycolytic activity in response to glucose suggests that Sfp1 is an upstream regulator that plays a role at the crossroads of ribosome biogenesis and central carbon metabolism.

\section{ACKNOWLEDGEMENTS}

This work was supported by the IOP-Genomics programme of Senter/Novem, and by the Netherlands Genomics Initiative (NGI) of the Netherlands Science Council (NWO). The authors wish to thank Marinka Almering and Erwin Suir for expert technical assistance, and Theo Knijnenburg for his expert contribution on microarray data mining. Marina Vai is specially thanked for thorough scientific discussions.

\section{REFERENCES}

Ahuatzi, D., Riera, A., Pelaez, R., Herrero, P. \& Moreno, F. (2007). Hxk2 regulates the phosphorylation state of Mig1 and therefore its nucleocytoplasmic distribution. J Biol Chem 282, 4485-4493.
Alberghina, L. \& Porro, D. (1993). Quantitative flow cytometry: analysis of protein distributions in budding yeast. A mini-review. Yeast 9, 815-823.

Alberghina, L., Smeraldi, C., Ranzi, B. M. \& Porro, D. (1998). Control by nutrients of growth and cell cycle progression in budding yeast, analyzed by double-tag flow cytometry. J Bacteriol 180, 3864-3872.

Berger, A. B., Decourty, L., Badis, G., Nehrbass, U., Jacquier, A. \& Gadal, O. (2007). Hmol is required for TOR-dependent regulation of ribosomal protein gene transcription. Mol Cell Biol 27, 8015-8026.

Boer, V. M., de Winde, J. H., Pronk, J. T. \& Piper, M. D. (2003). The genome-wide transcriptional responses of Saccharomyces cerevisiae grown on glucose in aerobic chemostat cultures limited for carbon, nitrogen, phosphorus, or sulfur. J Biol Chem 278, 3265-3274.

Cipollina, C., Alberghina, L., Porro, D. \& Vai, M. (2005). SFP1 is involved in cell size modulation in respiro-fermentative growth conditions. Yeast 22, 385-399.

Cipollina, C., van den Brink, J., Daran-Lapujade, P., Pronk, J. T., Vai, M. \& de Winde, J. H. (2008). Revisiting the role of yeast Sfp1 in ribosome biogenesis and cell size control: a chemostat study. Microbiology 154, 337-346.

Crespo, J. L. \& Hall, M. N. (2002). Elucidating TOR signaling and rapamycin action: lessons from Saccharomyces cerevisiae. Microbiol Mol Biol Rev 66, 579-591.

Daran-Lapujade, P., Rossell, S., van Gulik, W. M., Luttik, M. A., de Groot, M. J., Slijper, M., Heck, A. J., Daran, J. M., de Winde, J. H. \& other authors (2007). The fluxes through glycolytic enzymes in Saccharomyces cerevisiae are predominantly regulated at posttranscriptional levels. Proc Natl Acad Sci U S A 104, 15753-15758.

de Jong-Gubbels, P., Vanrolleghem, P., Heijnen, S., van Dijken, J. P. \& Pronk, J. T. (1995). Regulation of carbon metabolism in chemostat cultures of Saccharomyces cerevisiae grown on mixtures of glucose and ethanol. Yeast 11, 407-418.

DeSalle, L. M. \& Pagano, M. (2001). Regulation of the G1 to S transition by the ubiquitin pathway. FEBS Lett 490, 179-189.

Estruch, F. (2000). Stress-controlled transcription factors, stressinduced genes and stress tolerance in budding yeast. FEMS Microbiol Rev 24, 469-486.

Fatica, A. \& Tollervey, D. (2002). Making ribosomes. Curr Opin Cell Biol 14, 313-318.

Fingerman, I., Nagaraj, V., Norris, D. \& Vershon, A. K. (2003). Sfp1 plays a key role in yeast ribosome biogenesis. Eukaryot Cell 2, 1061-1068.

Flick, K., Chapman-Shimshoni, D., Stuart, D., Guaderrama, M. \& Wittenberg, C. (1998). Regulation of cell size by glucose is exerted via repression of the CLN1 promoter. Mol Cell Biol 18, 2492-2501.

Futcher, B. (1996). Cyclins and the wiring of the yeast cell cycle. Yeast 12, 1635-1646.

Gasch, A. P. \& Werner-Washburne, M. (2002). The genomics of yeast responses to environmental stress and starvation. Funct Integr Genomics 2, 181-192.

Griffioen, G., Laan, R. J., Mager, W. H. \& Planta, R. J. (1996). Ribosomal protein gene transcription in Saccharomyces cerevisiae shows a biphasic response to nutritional changes. Microbiology 142, 2279-2287.

Harbison, C. T., Gordon, D. B., Lee, T. I., Rinaldi, N. J., Macisaac, K. D., Danford, T. W., Hannett, N. M., Tagne, J. B., Reynolds, D. B. \& other authors (2004). Transcriptional regulatory code of a eukaryotic genome. Nature 431, 99-104.

Hoskisson, P. A. \& Hobbs, G. (2005). Continuous culture - making a comeback? Microbiology 151, 3153-3159. 
Hughes, J. D., Estep, P. W., Tavazoie, S. \& Church, G. M. (2000). Computational identification of cis-regulatory elements associated with groups of functionally related genes in Saccharomyces cerevisiae. J Mol Biol 296, 1205-1214.

Jansen, M. L., Diderich, J. A., Mashego, M., Hassane, A., de Winde, J. H., Daran-Lapujade, P. \& Pronk, J. T. (2005). Prolonged selection in aerobic, glucose-limited chemostat cultures of Saccharomyces cerevisiae causes a partial loss of glycolytic capacity. Microbiology 151, $1657-1669$.

Johnston, M. (1999). Feasting, fasting and fermenting. Glucose sensing in yeast and other cells. Trends Genet 15, 29-33.

Jorgensen, P., Nishikawa, J. L., Breitkreutz, B. J. \& Tyers, M. (2002), Systematic identification of pathways that couple cell growth and division in yeast. Science 297, 395-400.

Jorgensen, P., Rupes, I., Sharom, J. R., Schneper, L., Broach, J. R. \& Tyers, M. (2004). A dynamic transcriptional network communicates growth potential to ribosome synthesis and critical cell size. Genes Dev 18, 2491-2505.

Klein, C. \& Struhl, K. (1994). Protein kinase A mediates growthregulated expression of yeast ribosomal protein genes by modulating RAP1 transcriptional activity. Mol Cell Biol 14, 1920-1928.

Knijnenburg, T. A., de Winde, J. H., Daran, J. M., Daran-Lapujade, P., Pronk, J. T., Reinders, M. J. \& Wessels, L. F. (2007). Exploiting combinatorial cultivation conditions to infer transcriptional regulation. BMC Genomics 8, 25.

Knijnenburg, T. A., Wessels, L. F. A. \& Reinders, M. J. T. (2008). Creating gene set activity profiles with time-series expression data. IJBRA 4, 3 .

Kresnowati, M. T., van Winden, W. A., Almering, M. J., ten Pierick, A. Ras, C., Knijnenburg, T. A., Daran-Lapujade, P., Pronk, J. T., Heijnen, J. J. \& Daran, J. M. (2006). When transcriptome meets metabolome: fast cellular responses of yeast to sudden relief of glucose limitation. Mol Syst Biol 2, 49.

Laferte, A., Favry, E., Sentenac, A., Riva, M., Carles, C. \& Chedin, S. (2006). The transcriptional activity of RNA polymerase I is a key determinant for the level of all ribosome components. Genes Dev 20, 2030-2040.

Leek, J. T., Monsen, E., Dabney, A. R. \& Storey, J. D. (2006). EDGE: extraction and analysis of differential gene expression. Bioinformatics 22, 507-508.

Li, H., Tsang, C. K., Watkins, M., Bertram, P. G. \& Zheng, X. F. (2006) Nutrient regulates Tor 1 nuclear localization and association with rDNA promoter. Nature 442, 1058-1061.

Liko, D., Slattery, M. G. \& Heideman, W. (2007). Stb3 binds to ribosomal RNA processing element motifs that control transcriptional responses to growth in Saccharomyces cerevisiae. J Biol Chem 282, 26623-26628.

Marion, R. M., Regev, A., Segal, E., Barash, Y., Koller, D., Friedman, N. \& O'Shea, E. K. (2004). Sfp1 is a stress- and nutrient-sensitive regulator of ribosomal protein gene expression. Proc Natl Acad Sci U S A 101, 14315-14322.

Martin, D. E., Soulard, A. \& Hall, M. N. (2004). TOR regulates ribosomal protein gene expression via PKA and the Forkhead transcription factor FHL1. Cell 119, 969-979.

Mashego, M. R., van Gulik, W. M. \& Heijnen, J. J. (2007). Metabolome dynamic responses of Saccharomyces cerevisiae to simultaneous rapid perturbations in external electron acceptor and electron donor. FEMS Yeast Res 7, 48-66.

Moreno, F., Ahuatzi, D., Riera, A., Palomino, C. A. \& Herrero, P. (2005). Glucose sensing through the Hxk2-dependent signalling pathway. Biochem Soc Trans 33, 265-268.
Moss, T. (2004). At the crossroads of growth control; making ribosomal RNA. Curr Opin Genet Dev 14, 210-217.

Newcomb, L. L., Diderich, J. A., Slattery, M. G. \& Heideman, W. (2003). Glucose regulation of Saccharomyces cerevisiae cell cycle genes. Eukaryot Cell 2, 143-149.

Novick, A. \& Szilard, L. (1950). Experiments with the chemostat on spontaneous mutations of bacteria. Proc Natl Acad Sci U S A 36, 708719.

Ozcan, S., Dover, J., Rosenwald, A. G., Wolfl, S. \& Johnston, M. (1996). Two glucose transporters in Saccharomyces cerevisiae are glucose sensors that generate a signal for induction of gene expression. Proc Natl Acad Sci U S A 93, 12428-12432.

Piper, M. D., Daran-Lapujade, P., Bro, C., Regenberg, B., Knudsen, S., Nielsen, J. \& Pronk, J. T. (2002). Reproducibility of oligonucleotide microarray transcriptome analyses. An interlaboratory comparison using chemostat cultures of Saccharomyces cerevisiae. J Biol Chem 277, 37001-37008.

Popolo, L., Vanoni, M. \& Alberghina, L. (1982). Control of the yeast cell cycle by protein synthesis. Exp Cell Res 142, 69-78.

Postma, E., Kuiper, A., Tomasouw, W. F., Scheffers, W. A. \& van Dijken, J. P. (1989). Competition for glucose between the yeasts Saccharomyces cerevisiae and Candida utilis. Appl Environ Microbiol 55, 3214-3220.

Powers, T. \& Walter, P. (1999). Regulation of ribosome biogenesis by the rapamycin-sensitive TOR-signaling pathway in Saccharomyces cerevisiae. Mol Biol Cell 10, 987-1000.

Rolland, F., Wanke, V., Cauwenberg, L., Ma, P., Boles, E., Vanoni, M., de Winde, J. H., Thevelein, J. M. \& Winderickx, J. (2001). The role of hexose transport and phosphorylation in cAMP signalling in the yeast Saccharomyces cerevisiae. FEMS Yeast Res 1, 33-45.

Rolland, F., Winderickx, J. \& Thevelein, J. M. (2002). Glucose-sensing and -signalling mechanisms in yeast. FEMS Yeast Res 2, 183-201.

Schneider, B. L., Zhang, J., Markwardt, J., Tokiwa, G., Volpe, T., Honey, S. \& Futcher, B. (2004). Growth rate and cell size modulate the synthesis of, and requirement for, G1-phase cyclins at start. Mol Cell Biol 24, 10802-10813.

Tusher, V. G., Tibshirani, R. \& Chu, G. (2001). Significance analysis of microarrays applied to the ionizing radiation response. Proc Natl Acad Sci U S A 98, 5116-5121.

Urban, J., Soulard, A., Huber, A., Lippman, S., Mukhopadhyay, D., Deloche, O., Wanke, V., Anrather, D., Ammerer, G. \& other authors (2007). Sch9 is a major target of TORC1 in Saccharomyces cerevisiae. Mol Cell 26, 663-674.

van den Brink, J., Daran-Lapujade, P., Pronk, J. T. \& de Winde, J. H. (2008). New insights into the Saccharomyces cerevisiae fermentation switch: dynamic transcriptional response to anaerobicity and glucoseexcess. BMC Genomics 9, 100 .

van Helden, J., André, B. \& Collado-Vides, J. (2000). A web site for the computational analysis of yeast regulatory sequences. Yeast 16, $177-187$.

van Maris, A. J., Luttik, M. A., Winkler, A. A., van Dijken, J. P. \& Pronk, J. T. (2003). Overproduction of threonine aldolase circumvents the biosynthetic role of pyruvate decarboxylase in glucose-limited chemostat cultures of Saccharomyces cerevisiae. Appl Environ Microbiol 69, 2094-2099.

Vanoni, M., Vai, M., Popolo, L. \& Alberghina, L. (1983). Structural heterogeneity in populations of the budding yeast Saccharomyces cerevisiae. J Bacteriol 156, 1282-1291.

Verduyn, C., Postma, E., Scheffers, W. A. \& van Dijken, J. P. (1992). Effect of benzoic acid on metabolic fluxes in yeasts: a continuous- 
culture study on the regulation of respiration and alcoholic fermentation. Yeast 8, 501-517.

Warner, J. R. (1999). The economics of ribosome biosynthesis in yeast. Trends Biochem Sci 24, 437-440.

Westergaard, S. L., Oliveira, A. P., Bro, C., Olsson, L. \& Nielsen, J. (2007). A systems biology approach to study glucose repression in the yeast Saccharomyces cerevisiae. Biotechnol Bioeng 96, 134-145.
Xie, Y. \& Varshavsky, A. (2001). RPN4 is a ligand, substrate, and transcriptional regulator of the $26 \mathrm{~S}$ proteasome: a negative feedback circuit. Proc Natl Acad Sci U S A 98, 3056-3061.

Zurita-Martinez, S. A. \& Cardenas, M. E. (2005). Tor and cyclic AMPprotein kinase A: two parallel pathways regulating expression of genes required for cell growth. Eukaryot Cell 4, 63-71.

Edited by: H. A. B. Wösten 